\title{
Investigation of Water Penetration into Ceramides with Lateral Field Excitation Sensors
}

\author{
Joerg Fochtmann ${ }^{*}$, Anja Kaestner ${ }^{*}$, Ralf Lucklum*, Annett Schroeter ${ }^{\#}$, Reinhard H. H. Neubert ${ }^{\#}$ \\ * Otto-von-Guericke-University Magdeburg, Institute of Micro- and Sensor Systems, 39106 \\ Magdeburg, Germany \\ \# Martin-Luther-University Halle, Institute for Pharmaceutics and Biopharmaceutics, 06120 Halle, \\ Germany \\ Corresponding e-mail address: joerg.fochtmann@ovgu.de
}

\begin{abstract}
In this work we apply ceramide membranes using quartz crystal resonator sensors investigating water penetration into these membranes. The sensor response to pure Ceramide[AS] and Ceramide[AP] and mixtures with cholesterol immobilized onto the sensor surface and when immersed in deionized water is significantly different. After an annealing sequence to activate the ceramides, water immersion results in a resonance frequency shift of a classical Quartz Crystal Microbalance (QCM) primarily due to film mass increase caused by water absorption. Another sensor type, Lateral Field Excitation (LFE), shows qualitatively equal behavior: pure Ceramide[AS] or Ceramide[AP] do not display a diffusion related frequency shift whereas Ceramide[AS] mixed with cholesterol provides a significant change in resonance frequency and Q-factor.
\end{abstract}

\section{Introduction}

The upper human skin is made out of four layers: stratum corneum (SC), stratum granulosum, stratum spinosum, and stratum basale. Mainly, ceratinocytes built up these layers. Hereby the SC is mentioned as the most important barrier for water regulation and drug delivery [1]. Three basic compounds are present in the SC: ceramides, cholesterol and free fatty acids, whereas ceramides highly dominate this pattern, containing a sphingoid molecule bound to a fatty acid [1]. In [2] it is reported that about 9 different ceramides are present in human skin. Their characteristics vary in chain length and hydrophilic behavior realized by the placement of head groups.

On-line methods could provide direct information of drug delivery processes by sampling optical, mechanical or electrical properties of the membrane. Off-line investigation methods exist to investigate conformational changes before and after drugs were absorbed in the membrane, e. g. neutron diffraction studies [3]. In this paper, an artificial membrane containing various mixtures of the above mentioned materials imitate the SC. QCM and LFE sensors were coated with these membranes and then immersed into water. On-line monitoring using QCM and LFE sensors enable the observation of water penetration into these membranes.

LFE resonators provide information regarding resonant frequency dependence on electrical properties, i.e. relative permittivity $\varepsilon_{\mathrm{r}}$ and conductivity $\sigma$, in addition to mechanical properties of the ceramide membrane and a liquid above [4]. The large $\varepsilon_{\mathrm{r}}$ of water increases the overall permittivity of the immobilized film and therefore the LFE sensor can be expected to show a much higher resonance frequency response compared to QCM. Since LFE resonators can be understood as QCM with an additional electrical sensing parameter, both were used for the measurements and revealed comparable results. Another advantage of LFE compared to QCM is the bare quartz sensing surface (no top gold electrode). As a consequence, biochemical preparation procedures can be adopted from glass plates or electrode free sensor systems, for example electromagnetic piezoelectric acoustic sensors (EMPAS) [5], produced by Sheik et al.

\section{Background}

Based on the mentioned electrical sensing parameters, the applications for LFE should respond to altering electrical properties. The high dielectric constant $\varepsilon_{\text {r }}$ of water is well suited to affect the sensor's electrical field, since an electrical field is influenced by the permittivity of the ambient material [4]. Furthermore the piezoelectric conditions depend on free charges at the sensor surface. If those charges are not free, the electric field strength is affected, therefore the stiffness of the resonator material due changes in the piezoelectric conditions. Also the media's 
electric conductivity $\sigma$ affects the accumulation of surface charges, again resulting in a varied stiffness of the piezoelectric crystal [8]. Finally, the stiffness of the piezoelectric material alters the resonant behavior of the sensor, i. e. the impedance spectra as well as the resonance frequency are being affected [8]. The electrodes of LFE sensors act as an opened capacitor, hence consideration on the distance of the sensor surface to the water volume is necessary. Here, water mimics the dielectric material which can be recognized in a distance up to low millimeter range. Consequently, the measured impedance spectra should respond to the water penetration process.

The impedance analyzer calculates the complex impedance $\underline{Z}$ spectra for the sampled frequency range. The real part $G$ of the complex admittance $\underline{Y}$ is used, computed through $G=\operatorname{Re}\{\underline{Y}\}=\operatorname{Re}\{1 / \underline{Z}\}$ for further analysis. The maximum $G_{\max }$ of $G$ occurs at the series resonance frequency $f_{\text {res. }}$. Resonance frequency $f_{\text {res }}$ versus time $t$ as well as $G_{\max }$ versus $t$ were plotted and saved by the data acquisition computer showing the sensor responses to coatings and water penetration over time. Since complete spectra were obtained, half bandwidth $\mathrm{HBW}$ and quality factor $Q=f_{\text {res }} / \mathrm{HBW}$ could be obtained. The $\mathrm{HBW}$ is calculated by $\mathrm{HBW}=\left|f_{(G \max / 2)}-f_{\text {res }}\right|$. Q represents a measure for viscoelastic changes, as well as HBW does, in agreement with the acoustic load concept [6].

\section{Experimental Setup}

An in house developed impedance analyzer (ELISA150) as well as a commercial device (HP 4395A) were used to investigate the sensor behavior. Clamping of the crystal was done in a PTFE holder with two low force spring contacts, and high elastic O-rings on top and bottom, covered by a lid for static or flow measurements. The crystal holder fitted to both, the commercial and the in house system. Crystals (LapTech Precision, Bowmanville Canada) used had a diameter of $14 \mathrm{~mm}$, and gold electrodes with $7 \mathrm{~mm}$ diameter. Moreover, with a thickness of $330 \mu \mathrm{m}$ the resulting resonant frequencies were approx. $5 \mathrm{MHz}$ (C100 Mode) and approx. 5.06 MHz (C101) for
LFE and QCM $(166 \mu \mathrm{m})$ were $10 \mathrm{MHz}$. The sampling frequency ranges from $4.96 \mathrm{MHz}$ to 5.02 $\mathrm{MHz}(\mathrm{C} 100)$ and $5.045 \mathrm{MHz}$ to $5.08 \mathrm{MHz}$ (C101), the frequency resolution is $75 \mathrm{~Hz}$. Experiments were performed with a volume of $200 \mu \mathrm{l}$ poured onto the sensor which results in a $2.1 \mathrm{~mm}$ liquid column height. Either a plastic lid or parafilm was used as a sealing to avoid water evaporation.

\section{Chemicals and Preparation}

Ceramide[AS] (abbr.: CerAS) and ceramide[AP] (abbr.: CerAP), were provided by University Halle, Germany. Palmitic acid (abbr.: Palm), cholesterol (abbr.: Chol), methanol, ethanol, choloform, were obtained from the in house chemicals store (p.a. grade). Distilled water was produced in house.

The cleaning steps for the sensor were: 10 min soaping, rinsing with lukewarm water and deionized water, nitrogen stream drying, 10 min shaking in methanol, again nitrogen stream cleaning, $10 \mathrm{~min}$ nitrogen plasma etching. Contact angle measurements were done for plasma cleaned sensors. For clean surfaces, contact angles of less than $5^{\circ}$ were obtained, illustrating a very hydrophilic surface.

For model membranes, solutions with 10 $\mathrm{mg} / \mathrm{ml}$ of CerAS, or CerAP in solvent, containing three parts chloroform and one part methanol, were used as solely lipid solution. Mixed solutions containing lipid and cholesterol were prepared each component $5 \mathrm{mg} / \mathrm{ml}(1: 1$ mass). In consequence, the following solutions were used: CerAS, CerAP, CerAS:CerAP, CerAS:CerAP:Chol, CerAS:Chol, CerAP:Chol, CerAS:Chol:Palm, and CerAP:Chol:Palm.

The settings for spin-coating found to be appropriate with $10 \mathrm{sec}$ accelerate to 1200 rpm, keep the speed for $10 \mathrm{sec}$, and then decelerate $10 \mathrm{sec}$ to $0 \mathrm{rpm}$. The amounts poured onto the sensor surface equaled $50 \mu \mathrm{l}$, repeated three times, resulting in $150 \mu \mathrm{l}$ altogether. Before spin-coating the sensor was heated to $98{ }^{\circ} \mathrm{C}$, according to [3], to unfold the lipids. Here, CerAS unfolds at $98{ }^{\circ} \mathrm{C}$ and CerAP at $89{ }^{\circ} \mathrm{C}$. A final annealing step was used for rearrangement: $60 \min 98{ }^{\circ} \mathrm{C}, 100$ $\% \mathrm{RH} ; 15 \min \mathrm{RT} ; 30 \min 98^{\circ} \mathrm{C}, 100 \% \mathrm{RH} ; 15$ $\min \mathrm{RT} ; 30 \min 98^{\circ} \mathrm{C}, 100 \% \mathrm{RH}$. 


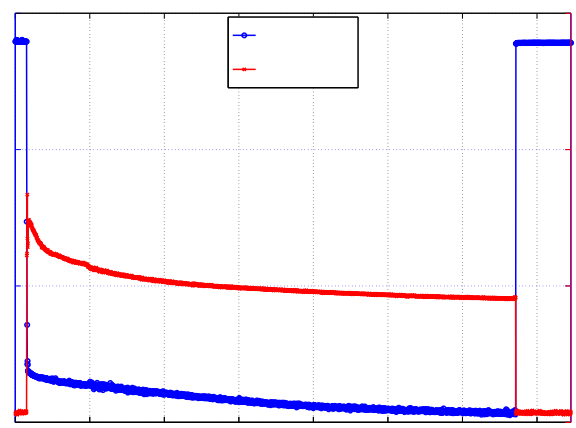

a)

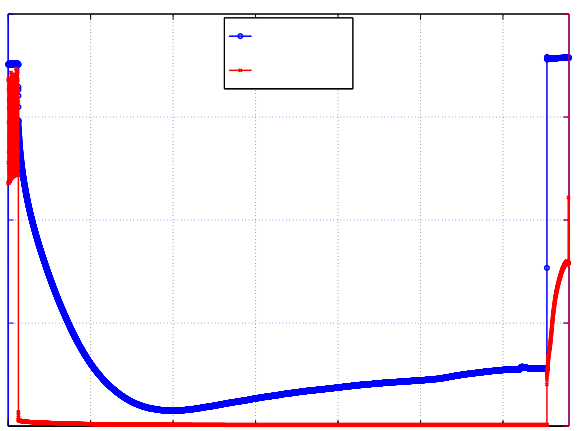

b)

Fig. 1: Sensor coated with CerAS:Chol and immersed in $200 \mu$ distilled water: a) LFE, b) QCM

\section{Results and Discussion}

The results show the water diffusion experiments into ceramide modified surfaces on LFE and were compared with coated QCM. We are using a two layer arrangement. The first layer represents the rigid film that is the ceramide mix. The second layer that is gaseous or liquid varies between air and water, respectively. The mass effect is overlaid by the acoustic effect, resulting in general acoustic impedance ZL. Exemplarily, Fig. 1a shows a typical resonance frequency shift (fmax) for a CerAS-Chol coated LFE sensor (C101) as well as the corresponding conductance maxima (Gmax). Two main shifts due to water immersion are observable: firstly, adding water results in $11.8 \mathrm{kHz}$ shift; secondly, a time dependent drift process occurs (approx. 1.5 $\mathrm{kHz}$ ). Comparing results for cholesterol and cholesterol free coatings in fig. 2a display, only CerAS-Chol provides such high values. Every other combination is less influenced by the presence of distilled water. Diffusion of water accompanied by a change of the electrical field strength results in altered resonant conditions, and hence reduces the resonant frequency.

From Fig. 2a it becomes clear, that the Qfactor change in addition to the resonance frequency shift provides significant information on interactions. Furthermore, Fig. 2a displays the different sensor responses to mixtures applied, and immersed in $200 \mu \mathrm{l}$ deionized water. The strong Q-factor change of CerASChol compared to little values of other mixtures let us assume that mixtures of both chemicals strongly support the diffusion process.

QCM resonators coated with CerAS-Chol were used to evaluate the obtained LFE behavior. In terms of thicknesses, for a $5-\mathrm{MHz}-$ QCM a shift of $747 \mathrm{~Hz}$ results that is close to the LFE shift of $737 \mathrm{~Hz}$. Calculating the thicknesses from the mentioned frequency shifts according to Sauerbrey equation, both, QCM and LFE sensor, are coated similarly. Fig. $1 \mathrm{~b}$ depicts a sampled diagram similar to LFE, although the resonant frequency reaches a minimum at 20,000 seconds already, and afterwards increases again. Pouring water onto the QCM surface produces a frequency shift of $2,710 \mathrm{~Hz}$. Hereafter a frequency drift of 14,000 $\mathrm{Hz}$ takes place, due to diffusion. Clearly, the initial shift generated by water immersion for QCM with $\Delta$ fres, $Q C M=2,710 \mathrm{~Hz}$ (at $10 \mathrm{MHz}$ ) is very little compared to $\Delta$ fres, $L F E=11.8 \mathrm{kHz}$. Obviously it is produced by the electric parameter sensitivity of LFE to water's relative permittivity $\varepsilon r$.

Half bandwidth values for QCM and LFE resonators coated with CerAS-Chol are displayed in Fig. 2b. Water immersion experiments were done twice in a row, with no remove of sensor meanwhile. The sensor was annealed for two hours and again $200 \mu \mathrm{l}$ distilled water were poured onto the surface. We have to consider a completely different pattern. In contrast to first immersion, no frequency shift governed by diffusion is present for the second immersion. We assume complete molecular rearrangements during annealing occur. Indeed, literature supports this assumption. In [7] it is explained by the structure of ceramides, where X-ray diffraction studies show a conformation change due to temperature treatment. One assumption is that the hydrating process results in a hexagonal ceramide-cholesterol arrangement. The structure keeps after annealing. Additionally, free bonding places may not exist anymore, in consequence water molecules cannot penetrate into the surface anymore. 


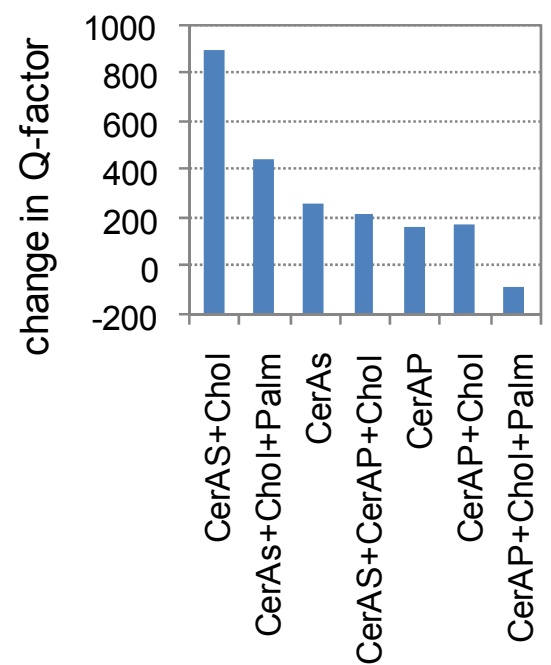

a)

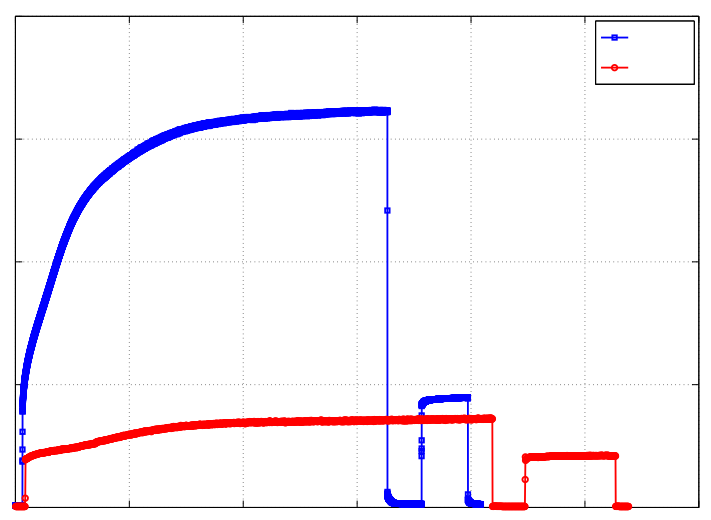

b)

Fig. 2: a) Change of Q-factor for different mixtures of ceramides/cholesterol/palmitic acid on LFE sensors; $b)$ Half bandwidth $B(H B W)$ versus time for QCM and LFE sensor coated with Ceramide[AS] and cholesterol, first sequence: immersed in $200 \mu$ l distilled water; second sequence: water removed; third sequence: again $200 \mu l$ distilled water added; fourth sequence: water removed again;

\section{Conclusion}

Water diffusion can be observed both with QCM and LFE sensor types, since both sample mechanical parameters of the applied media. LFE sensor sensitivity to highpermittivity fluids is strong compared to QCM, hence the sensor response can be different to QCM depending on functional layer parameters. Both effects can be used in drug delivery research, with special care to the delivered drug in terms of electrical properties $\sigma$ and $\varepsilon_{r}$. Generally, biomolecules or biochemicals possess low conductivity (less than

\section{References}

[1] J. A. Bouwstra et al, J. Invest. Dermatol. 118 (4), (2002) 606-617.

[2] M. W. de Jager et al, Journal of Lipid Research, Volume 46 (2005) 2649-2656.

[3] A. Schroeter et al, Biochimica et Biophysica Acta 1788 (2009) 2194-2203.

[4] U. Hempel et al, Meas. Sci. Technol. 19 (2008) 055201.

[5] S. Sheikh et al, Chem. Sci. 1 (2010) 271275.

[6] R. Lucklum, P. Hauptmann, Sensors and Actuators B 70 (2000) 30-36.

[7] S. Raudenkolb, Chemistry and Physics of Lipids Vol. 136, Issue 1, (2005) 13-22.

[8] J. Fochtmann et al, Sensors Actuators B 151 (2011) in print. $\left.10^{-10} \mathrm{~S} / \mathrm{cm}^{2}\right)$ and low dielectric constants $\left(\varepsilon_{\mathrm{r}}=2\right.$ to 3). In order to gain significant sensor responses, one or both of those parameters need to alter or penetration of those molecules must be accompanied by water diffusion. Additional investigations have to be done with other ceramides and mixtures, to deepen their influence to water diffusion and drug delivery.

\section{Acknowledgements}

One of the authors wants to thank Sonia Sheikh, Christophe Blaszykowski and Mike Thompson from University of Toronto for their support in surface modification techniques. 